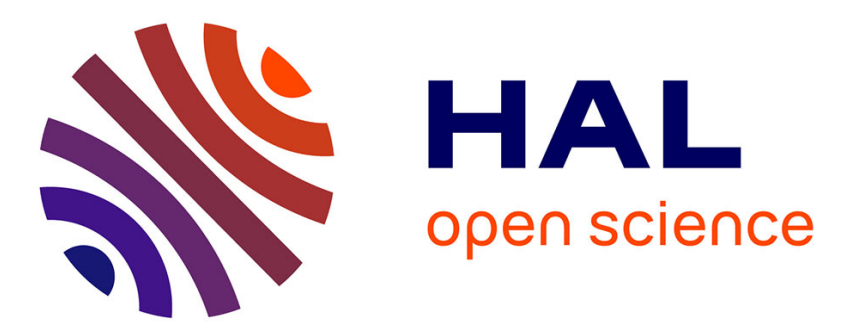

\title{
Les services urbains en réseau dans les pays arabes: diversité des réformes libérales et de leurs effets territoriaux
}

Éric Verdeil

\section{- To cite this version:}

Éric Verdeil. Les services urbains en réseau dans les pays arabes : diversité des réformes libérales et de leurs effets territoriaux: Introduction au dossier de Géocarrefour. Géocarrefour - Revue de géographie de Lyon, 2010, 85 (2), pp.99-109. 10.4000/geocarrefour.7774 . halshs-00489908

\section{HAL Id: halshs-00489908 \\ https://shs.hal.science/halshs-00489908}

Submitted on 7 Jun 2010

HAL is a multi-disciplinary open access archive for the deposit and dissemination of scientific research documents, whether they are published or not. The documents may come from teaching and research institutions in France or abroad, or from public or private research centers.
L'archive ouverte pluridisciplinaire HAL, est destinée au dépôt et à la diffusion de documents scientifiques de niveau recherche, publiés ou non, émanant des établissements d'enseignement et de recherche français ou étrangers, des laboratoires publics ou privés. 


\section{Les services urbains en réseau dans les pays arabes : diversité des réformes libérales et de leurs effets territoriaux}

Introduction du dossier de Géocarrefour (n85-2)

Eric VERDEIL

Université de Lyon

UMR Environnement Ville Société

eric.verdeil@normalesup.org

Résumé

Cet article constitue l'introduction du dossier sur les services urbains en réseau dans les pays arabes. Il propose d'abord une mise en perspective de leur situation par rapport aux autres pays en développement. Il apparaît que les PPP, figures majeures de ces réformes, ont relativement moins concerné les pays arabes. Les tensions géopolitiques régionales et la crainte de troubles en cas de remise en cause du contrat social entre les régimes et les populations apparaissent comme des facteurs de frein à ce type de réforme. Mais en complément des PPP, les réformes territoriales sont aussi un vecteur de transformation de la gestion des services urbains. Mais les exemples de décentralisation s'accompagnent de déconcentration et de reconcentration. Les arrangements avec les opérateurs informels ou petits opérateurs marchands, vus parfois comme une solution possible, ne débouchent pas, dans le monde arabe, sur une institutionnalisation. L'ensemble de ces transformations, souvent sur un mode expérimental, aboutit à une imbrication de situations territoriales souvent très différenciés, résultat inattendu de ces réformes.

Mots-clés : réforme, services urbains en réseau, infrastructure, décentralisation, pays arabes, villes en développement, Liban, Jordanie, Egypte, Maroc, Tunisie

\section{Les réformes des services urbains dans les villes en développement}

Les dysfonctionnements des services publics de base, comme l'eau, l'assainissement, l'électricité ou les déchets, constituent l'un des signes distinctifs majeurs des villes en développement par rapport à celles des pays industrialisés. Les inégalités territoriales de desserte sont à la fois le reflet et le vecteur de la pauvreté de larges segments de la population urbaine, et sont bien souvent également cruellement accusées entre villes et campagnes. De ce fait, l'amélioration de ces services de base est conçue comme un des leviers des stratégies de développement et d'atténuation de la pauvreté, en lien avec d'autres actions comme l'amélioration de la tenure foncière (Durand-Lasserve, 2004).

Depuis une vingtaine d'années, la réalisation de cette ambition a été entreprise dans un contexte marqué par les politiques d'ajustement structurel et largement dominé par l'agenda néolibéral. Conjointement portées par les élites politiques nationales et les représentants des bailleurs de fonds 
internationaux, les réformes des services publics en réseau combinent diverses mesures incluant la marchandisation, l'arrêt ou la limitation des subventions tarifaires afin que le prix des services reflète les coûts engagés, l'introduction d'une gestion inspirée des principes du secteur privée voire mise en œuvre par des opérateurs privés, etc. Les travaux de recherche dans ce domaine se sont particulièrement concentrés sur deux axes : les évolutions du secteur de l'eau potable, perçu comme le plus symbolique en raison de son caractère vital, et la participation à ce processus de multinationales spécialisées dans ce secteur, notamment françaises (voir par exemple SchneierMadanes 2003).

Le débat, en partie idéologique, sur l'efficacité des solutions inspirées du paradigme néolibéral a largement structuré la recherche et continue de le faire (Gassner et al. 2009). Dans le champ de la recherche urbaine, l'ouvrage Splinterring Urbanism a constitué depuis une dizaine d'années une référence dans les débats (Marvin, Graham 2001). Ses auteurs y analysent les réformes néolibérales dans le domaine des services urbains en réseau qui remettent en cause ce qu'ils nomment l'idéal moderne du service universel, caractéristique de la période du Welfare State. Ils affirment que ces transformations entraînent une fragmentation sociale et urbaine accrue. Cette conclusion s'appliquerait non seulement au monde américain et ouest-européen mais également aux « pays du Sud ».

De nombreuses recherches ont discuté, approfondi ou nuancé les conclusions de cet ouvrage. En particulier pour les pays du Sud, des travaux récents lui ont apporté de sérieuses objections (Coutard, 2008 propose notamment une instructive synthèse de ces critiques). Un premier problème réside dans la mise en évidence des décalages par rapport au standard du service universel. Si la situation des pays industrialisés peut, dans certains cas, s'analyser comme une régression par rapport à cette référence, telle n'est pas la situation de nombreux pays du Sud où la norme du service universel n'a jamais été effective, voire a constitué un modèle " parodique ", comme le montre S. Jaglin (2005) à propos de l'Afrique sub-saharienne. Marvin et Graham (2001) font du unbundling (la désintégration des entreprises du secteur) des opérateurs des services urbains, en lien avec la mise en place d'une logique de marchandisation accrue, l'un des principaux marqueurs de la transition en cours. Mais dans les pays du Sud, le sens des évolutions est bien plus complexe à caractériser puisque en raison même de l'inachèvement du modèle des services publics universels, on observe une grande diversité en termes de fournisseurs de services, dont une partie sont dans le secteur informel, et en termes de qualité. D'une part, l'introduction de nouveaux partenariats publics-privés et les réformes institutionnelles de ces secteurs ajoutent de la complexité plus qu'ils ne constituent un mouvement d'une situation d'intégration fonctionnelle marquée par la dominance des acteurs publics vers une situation où les acteurs privés auraient le dessus. D'autre part, il convient aussi de souligner les limites du mouvement de réforme malgré un certain nombre d'exemples spectaculaires (Bakker 2003). Souvent, en dehors de quelques capitales ou villes importantes qui concentrent l'attention, les financements des organisations internationales et les appétits des multinationales ou des groupes capitalistes locaux, et en particulier dans les villes petites et moyennes, on ne constate pas de transformation notable de l'ancien modèle de gestion (pour l'Afrique sub-saharienne, Jaglin 2005).

Ces travaux invitent donc à dépasser la seule thématique de la privatisation, ou plus précisément de réformes introduisant des partenariats public-privé (PPP), pour replacer ces dernières dans un ensemble beaucoup plus vaste, incluant également des réformes du secteur public sans PPP, ainsi qu'une redistribution des responsabilités et des pratiques dans la gestion des services publics entre 
acteurs publics centraux et locaux (déconcentration et/ou décentralisation). Cette ambition rejoint une autre initiative éditoriale concomitante de présent dossier (Jaglin, Zérah, à paraître). En outre, les temporalités propres de ces réformes doivent être prises en considération. A l'idée d'une transformation radicale, opérée une fois pour toutes, que partisans et opposants des PPP promettent ou refusent au début de la réforme, il convient d'opposer la réalité de processus plus complexes, faits de détours, de moments de blocage et de réorientation, qui modifient la signification de telles évolutions. Dans la prise en compte de cette complexité, un point attire particulièrement l'attention des chercheurs : il s'agit des modalités d'articulation entre les opérateurs publics ou privés officiels et les acteurs du secteur longtemps considéré comme informel ou illégal, qu'on tend à qualifier aujourd'hui d'artisanal (pour les transports en particulier, voir Godard 2002) ou plus largement de petit secteur marchand (Botton, Blanc, 2010). Longtemps considéré au mieux comme transitoire sinon parasitaire, ce dernier apparait aux yeux de certains gestionnaires et de certains chercheurs comme une partie de la solution à l'universalisation du service public (Jaglin, Zérah, à paraître).

\section{Les services urbains dans les pays arabes}

Nous proposons ici d'examiner ces questions à partir d'un ensemble de cas sélectionnés en fonction d'un double critère, géographique et sectoriel. Le dossier vise à rassembler des informations sur les réformes en cours dans les pays arabes. En effet, en comparaison de l'Amérique latine (SchneierMadanes 2003), de l'Afrique (Jaglin 2005) ou de l'Asie du Sud et de Sud-Est (par ex. Zérah 1999, Bakker 2007), cette zone géographique est sous-représentée dans la littérature sur les réformes des services urbains. Un premier axe de questionnement consiste donc à interroger cette originalité, à travers un examen quantitatif de la situation ainsi que par une réflexion sur les raisons susceptibles de l'expliquer.

En tout état de cause, la question de la libéralisation du secteur des services urbains n'a pourtant pas été absente de l'agenda dans ces pays. Dès 1999, un important colloque organisé par le laboratoire SEDET y était consacrée (Chanson-Jabeur et al., 2001). Les études de cas sur de multiples secteurs d'activité (eau, logement, transport, éducation et santé) y dessinaient l'image d'une puissante et irrésistible vague libérale qui, après les transports urbains, s'attaquait au secteur de l'eau potable (Semmoud 2001), qui a par la suite focalisé l'attention des chercheurs (Deboulet 2000b). Le Maroc représente ici un pionnier puisque depuis 1997, plusieurs délégations de service (incluant l'eau potable, l'assainissement et l'électricité) ont été conclues à Casablanca, Rabat-Salé, Tanger et Tétouan et qui ont, de fait, fait l'objet de nombreuses analyses (Haoues-Jouve 1999 ; Allain ElMansouri 2001 ; De Miras, Le Tellier, 2005 ; etc.). D’autres expériences, au moindre retentissement, ont concerné Amman (Darmame et Potter 2009 ; les autres sur Amman), Tripoli au Liban (Yousfi 2006) ou encore la Palestine (Signoles 2004 et $2010^{* 1}$ ). Ces expériences sont demeurées limitées, leur bilan a été critiqué notamment par les autorités publiques clientes et, hormis au Maroc, les entreprises multinationales impliquées tendent aujourd'hui à se retirer ${ }^{2}$. Au-delà du bilan de ces opérations et des raisons des déceptions qu'elles ont suscitées, il faut alors se demander ce qui reste des réformes une décennie ou plus après leur entrée en vigueur.

\footnotetext{
${ }^{1}$ Nous indiquons par une étoile $(*)$ les références constitutives de ce dossier.

${ }^{2}$ Pour un bilan faisant une certaine place à l'autosatisfaction, voir Martinand, 2009 (étude datant de 20062007).
} 
L'objet de ce numéro est également de porter l'analyse au-delà du seul secteur de l'eau qui, dans les études des géographes et urbanistes, est ultra-dominant ${ }^{3}$ pour le monde arabe comme ailleurs. L'entrée par plusieurs types de réseaux d'infrastructure permet de prendre en compte plusieurs paramètres de l'analyse, notamment les contraintes techniques et les enjeux économiques liés à l'organisation du service, de même que ses particularités institutionnelles, par exemple en termes d'échelon territorial de gestion. La gestion de chaque secteur s'inscrit en effet dans un complexe socio-politique localisé, dimension qu'une vision monosectorielle risque d'occulter de l'analyse. A travers cette diversité d'études de cas, l'objectif est d'interroger les contours du contrat social implicite passé, depuis la période des indépendances, entre l'Etat et ses citoyens, en particulier par le biais des mécanismes de rente. Grâce aux dividendes du pétrole (parfois indirects dans les pays non pétroliers exportateurs de main d'ouvre) ou de l'aide internationale (Egypte, Jordanie en particulier), les Etats ont mis sur pied et financé des services publics, peu ou pas tarifés aux usagers. Dans une perspective d'économie politique, ces services s'inscrivent dans un échange entre la population et l'Etat, et sont vus comme un instrument de construction et de reproduction de la légitimité de ces régimes (Catusse, 2006 pour une synthèse de ces questions). A la suite de divers travaux (Tomeira, 2008) nous postulons que le pilotage des réformes varie d'un secteur à l'autre en fonction des coalitions d'intérêts en cause mais du risque social et politique. Par ailleurs, et plus globalement, les tensions géopolitiques très fortes qui affectent des pays comme le Liban et les Territoires palestiniens pèsent sur le processus réformiste.

En dépassant le seul débat sur l'introduction des PPP dans les services en réseau dans les pays arabes, nous faisons en fait l'hypothèse d'une pluralité de processus à l'œuvre. Constat banal mais qui a une implication territoriale forte : au lieu de chercher à identifier une tendance commune et un modèle d'organisation spatiale repérable dans divers contextes urbains ou régionaux (par exemple, une ségrégation spatiale accrue), nous postulons une différenciation multiforme, entre pays et, ce qui est potentiellement plus surprenant, entre villes voire même à l'intérieur d'une même ville ou agglomération.

Le matériau réuni pour aborder ce jeu d'hypothèses est quelque peu réduit et les conclusions qu'on peut en tirer ont donc un caractère exploratoire. Deux des articles (Signoles 2010, Allès 2010), auquel on peut rattacher un troisième (Gabillet 2010) sont issus du programme de recherche TANMIA regroupant notamment politologues et géographes, qui porte sur « Le développement et la fabrique de l'action publique dans le monde arabe », et auquel participe également le coordinateur de ce dossier. L'appel à contribution invitait à compléter ces regards. L'article de Bénédicte Florin, géographe, le fait à partir d'une entrée privilégiant les acteurs du secteur informel, en l'occurrence les chiffonniers du Caire (Florin 2010). Contrairement à ces articles qui combinent des analyses fines et localisées des jeux d'acteurs et de leurs inscriptions territoriales, le texte de Claude de Miras, économiste à l'IRD en appui à la direction de la décentralisation du ministère marocain de l'Intérieur, propose une description des transformations du secteur de l'eau et de l'assainissement à l'aune des statistiques consolidées des bilans financiers et opérationnels de ce secteur de l'action publique. Le dossier s'accompagne de 4 recensions portant sur l'urbanisme dans le monde arabe, qui illustrent le tournant libéral en cours dans les grandes villes, mais aussi les tendances plus complexes et moins spectaculaires de l'aménagement urbain au quotidien, dans les villes moyennes d'Egypte comme dans les périphéries maghrébines. Cet article introductif développe les principaux thèmes ci-dessus

\footnotetext{
${ }^{3}$ En économie, le secteur des télécommunications et celui de l'énergie sont beaucoup mieux représentés.
} 
esquissés, en s'appuyant sur les contributions et d'autres travaux récents ${ }^{4}$. Un point liminaire propose un cadrage quantitatif du mouvement des réformes à partir d'indicateurs fournis par la Banque mondiale.

\section{Public-privé : diversité et inflexion des trajectoires}

La région du Moyen-Orient et de l'Afrique du Nord, dans laquelle se situent les pays arabes étudiés dans ce dossier, est loin de représenter une région pilote du point de vue de la mise en œuvre de PPP. On utilise ici les statistiques compilées dans la base de données de la Banque mondiale sur la participation du secteur privé dans les infrastructures (PPI, Private Participation in Infrastructure Project), qui concerne uniquement les pays à bas et moyen revenu et les secteurs dits monopolistiques (énergie, eau, transports et télécommunications) ${ }^{5}$. De ces données ressortent deux tendances. Tout d'abord, en termes globaux sur la période pour laquelle les informations sont disponibles, et tous secteurs confondus, la région Moyen-Orient et Afrique du Nord n'attire que 123 projets, dont seulement 9 et 19 pour l'eau et assainissement et l'énergie (essentiellement le secteur électrique). Cela représente en moyenne 10 projets par pays, à peine plus que les 9 en Afrique Subsaharienne, elle aussi largement délaissée par les investissements internationaux dans les infrastructures. A l'inverse, l'Asie orientale et le Pacifique, l'Asie du Sud et l'Amérique latine le nombre de PPP dépasse les 45 par pays.

La figure 1 montre que la région Moyen-Orient et Afrique du Nord présente d'autre part un décalage temporel important par rapport aux autres régions, avec un démarrage sensible uniquement à partir de la fin des années 1990 et des oscillations beaucoup moins prononcées ensuite, en comparaison des variations puissantes observables dans les autres régions (pics de l'Europe orientale et de l'Amérique latine en 1993 et 1997-1998, pic en 1997 puis en 2007 pour l'Asie orientale et pacifique, mouvement comparable pour l'Asie du Sud). Ces quelques données soulignent clairement que la région reste donc relativement à l'écart de la grande vague de " privatisations » qui a touché les pays en développement depuis les années 1990 . Semmoud (2001, p. 669) notait certes ce point mais en anticipant une montée en puissance du phénomène qui, finalement, ne s'est pas réellement produit. Figure 1 : évolution du nombre de PPP par grandes régions (tous secteurs confondus)

\footnotetext{
${ }^{4}$ Ces réflexions sont nourries des discussions tenues dans le cadre de l'ANR TANMIA (2008-2010) et lors des séminaires "Services en réseau dans les villes en développement » (SERVED) organisé par Luisa Moretto, Sylvy Jaglin et Aymeric Blanc au nom du Laboratoire Techniques Territoires Société (Ecole des Ponts et Chaussées) et de l'Agence française de développement (2010).

${ }^{5}$ En sont donc exclus les pays arabes du Golfe ; ainsi que le secteur des déchets solides, dont traite néanmoins ce dossier.
} 


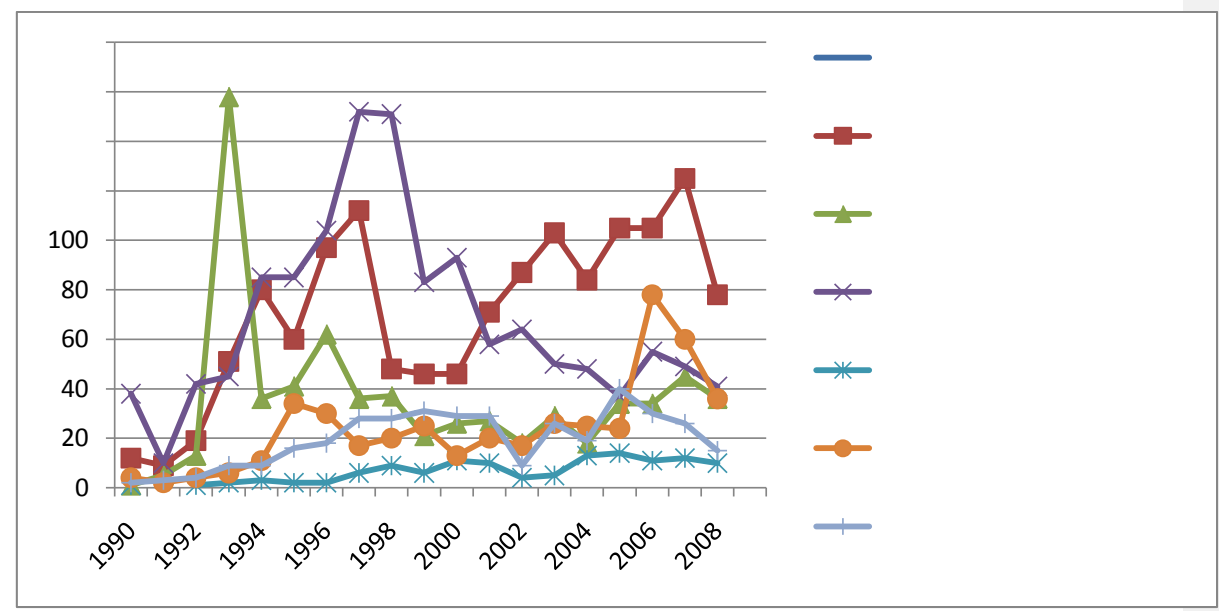

Source: World Bank and PPIAF, PPI Project Database. (http://ppi.worldbank.org) Date: mai 2010

Tableau 1 : Partenariats public-privé dans les pays en développement par grande région

\begin{tabular}{|c|c|c|c|c|c|c|}
\hline Région ONU & $\begin{array}{l}\text { Nombre de } \\
\text { pays non } \\
\text { documenté }\end{array}$ & $\begin{array}{l}\text { Nombre de } \\
\text { pays par } \\
\text { zone }\end{array}$ & $\begin{array}{l}\text { Nombre de } \\
\text { projets }\end{array}$ & $\begin{array}{l}\text { Nombre } \\
\text { moyen de } \\
\text { projet }\end{array}$ & $\begin{array}{l}\text { dont projet Eau } \\
\text { et assainissement }\end{array}$ & $\begin{array}{l}\text { dont projet } \\
\text { électricité }\end{array}$ \\
\hline $\begin{array}{r}\text { Asie orientale } \\
\text { et Pacifique }\end{array}$ & 3 & 23 & 1342 & 67 & 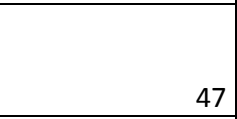 & 90 \\
\hline $\begin{array}{r}\text { Europe et Asie } \\
\text { centrale } \\
\end{array}$ & 0 & 24 & 678 & 28 & 31 & 68 \\
\hline $\begin{array}{r}\text { Amérique } \\
\text { latine et } \\
\text { Caraïbes }\end{array}$ & 0 & 29 & 1299 & 45 & 79 & 141 \\
\hline $\begin{array}{r}\text { Moyen-Orient } \\
\text { et Afrique du } \\
\text { Nord } \\
\end{array}$ & 1 & 13 & 123 & 10 & 9 & 19 \\
\hline Asie du Sud & 0 & 8 & 452 & 57 & 6 & 46 \\
\hline $\begin{array}{r}\text { Afrique } \\
\text { Subsaharienne } \\
\end{array}$ & 3 & 47 & 379 & 9 & 24 & 72 \\
\hline & & & 4273 & & 196 & 436 \\
\hline
\end{tabular}

Source: World Bank and PPIAF, PPI Project Database. (http://ppi.worldbank.org) Date: mai 2010

Une analyse chronologique et par pays de la mise en place des PPP dans les domaines de l'eau et assainissement et de l'énergie montre une légère accélération dans les années récentes, en fait très largement imputable à l'Algérie qui a lancé 10 PPP entre 2004 et 2008 (tableau 2). Aucun autre pays n'en compte d'ailleurs autant que cette dernière et, à l'exception de la Jordanie et de l'Irak, on trouve très peu de nouvelles opérations. L'essentiel des derniers contrats (sauf dans le cas algérien) concerne le secteur de l'énergie où les PPP se révèlent moins conflictuels.

Tableau 2 : les PPP dans les domaines de l'énergie (électricité) et de l'eau et assainissement dans les pays arabes 


\begin{tabular}{|l|c|c|c|c|c|c|c|c|c|c|}
\hline Année & Algérie & Egypte & Irak & Jordanie & Liban & Maroc & Tunisie & $\begin{array}{c}\text { Terr. } \\
\text { Palest. }\end{array}$ & Yémen & Total \\
\hline 1992 & & 1 & & & & & & & & 1 \\
\hline 1993 & 1 & & & & & 1 & 1 & & & 3 \\
\hline 1996 & & & & & & & & 1 & & 1 \\
\hline 1997 & & & & & & 2 & & & & 2 \\
\hline 1998 & & 1 & & & & 1 & 1 & & & 3 \\
\hline 1999 & & 1 & & 1 & & & 1 & 1 & & 4 \\
\hline 2000 & & & & & & & & 1 & & 1 \\
\hline 2001 & & 1 & & & & 1 & & & & 2 \\
\hline 2002 & & & & & 1 & & 1 & & & 2 \\
\hline 2003 & & & & 1 & & 1 & & & & 2 \\
\hline 2004 & 1 & & & & & & & & & 1 \\
\hline 2005 & 3 & & & & & & & & & 3 \\
\hline 2006 & 2 & & 1 & & & & & & 1 & 4 \\
\hline 2007 & 2 & 1 & 1 & 1 & & & & & & 5 \\
\hline 2008 & 2 & & & 1 & & & & & & 3 \\
\hline Total & 11 & $\mathbf{5}$ & $\mathbf{2}$ & $\mathbf{4}$ & $\mathbf{1}$ & $\mathbf{6}$ & $\mathbf{4}$ & $\mathbf{3}$ & $\mathbf{1}$ & $\mathbf{3 7}$ \\
\hline
\end{tabular}

Source: World Bank and PPIAF, PPI Project Database. (http://ppi.worldbank.org) Date: mai 2010

Ces données, à l'échelle globale comme à celle de la région de référence, invitent donc à considérer la question des réformes d'une manière plus large que les seuls PPP qui restent, malgré leur caractère spectaculaire, en nombre limité et concentrés dans les grandes métropoles (Bakker, 2010). La marchandisation des services urbains peut tout autant être mise en œuvre dans le cas d'entreprises publiques que par le biais d'un opérateur privé. Une comparaison récente des performances des PPP et des opérateurs publics du secteur menée par le PPIAF (Public-Private Infrastructure Advisory Facility) inclut ainsi dans son échantillon de référence approximativement trois fois plus d'opérateurs publics que de PPP (Gassner, Popov, Pushak, 2009).

L'exemple marocain présenté dans ce dossier par Claude de Miras (2010*) constitue une intéressante - quoique singulière par rapport aux autres pays arabes - illustration des évolutions de ce mouvement réformiste. D'une part, le royaume chérifien, après avoir concédé la gestion des régies des grandes villes du nord du pays, de Casablanca à Tanger, n'a pas poursuivi sur cette lancée après 2004. D'autre part, la gestion des secteurs de l'eau potable et de l'assainissement se caractérise par la persistance d'importantes subventions croisées entre secteurs (l'électricité finançant l'eau potable et l'assainissement) et entre espaces urbains et zones rurales. Toutefois ces péréquations s'effectuent en limitant drastiquement les subventions de fonctionnement de la part de l'Etat et en facturant ces services de manière à équilibrer le cout global de revient. En revanche, illustration d'un retour de balancier vers une perspective moins orthodoxe par rapport aux principes néolibéraux prévalant précédemment, les financements publics sont à nouveaux sollicités pour réaliser les investissements dans deux domaines où la mise à niveau est jugée prioritaire : l'environnement, à travers l'extension de l'assainissement ; et la pauvreté urbaine, à travers l'extension subventionnée des services essentiels. Sur ce dernier point, toutefois, $C$. de Miras pointe les difficiles contraintes financières auxquels l'Etat marocain doit faire face, et sa dépendance face aux bailleurs de fonds étrangers. 


\section{PPP et réformes des services urbains : de quelques facteurs bloquants}

L'analyse des inflexions dans la mise en œuvre des réformes du secteur des services urbains implique la prise en compte de différents facteurs. Au niveau global, les crises financières en Asie en 1997 et en Argentine en 2002 ont souligné aux yeux des multinationales présentes sur ces marchés les risques de ces contrats et elles ont revu leurs investissements à la baisse. La rentabilité espérée, en particulier par l'élargissement de la desserte auprès des ménages pauvres, n’a pas été au rendezvous (Bakker, 2009). Le discours de la Banque mondiale et des organisations internationales n'est plus aussi fortement en faveur des PPP. Toutefois, dans le cadre de ce dossier, ce ne sont pas ces questions qui émergent mais plutôt les conjonctures locales qui pèsent sur le calendrier des réformes et la définition du contenu de ces dernières.

A ce titre, un premier élément pèse lourdement sur ces enjeux : les tensions géopolitiques que connaissent certains Etats. Certes, I'Irak post-Saddam Hussein sous tutelle américaine se distingue par un recours aux PPP (tableau 2). On manque hélas de travaux sur cette question. En revanche, dans les Territoires palestiniens, où la mise en œuvre de PPP avait commencé à la fin des années 1990, l'Intifada d'Al Aqsa et la dégradation de la conjoncture politique interne a conduit au désengagement des firmes internationales impliquées, remplacées par des firmes privées locales (Signoles 2010*). Au Liban, la seule expérience de PPP dans le domaine de l'eau à Tripoli, lancée en 2002, a été interrompue en 2007. Si les facteurs proprement tripolitains ont fortement joué, la dégradation du climat politique après la guerre de 2006 a également contribué au blocage de la situation (Martinand 2009, p.50-52 ; Verdeil, Féré, Scherrer 2009). Toujours au Liban, la crise électrique, qui a culminé dans une émeute sanglante en janvier 2008, est l'un des catalyseurs des oppositions et tensions internes (Verdeil, 2009). Cela explique le blocage des réformes concernant l'électricité, pour laquelle des PPP voire une privatisation complète sont envisagés depuis 2002, et la pérennisation d'une situation de pénurie qui rend indispensable le recours à un système de générateurs et de mini-réseaux électriques (Gabillet, 2010*).

Un deuxième élément, en partie connexe au précédent, contribue à freiner ou à limiter le recours aux PPP : le risque est en effet de remettre en cause, à travers une politique de marchandisation des services essentiels, des contrats sociaux établis en longue date entre les gouvernements et leurs populations. La distribution d'eau potable ou la fourniture d'électricité sont en effet, dans nombre de pays, largement subventionnés. Dans divers cas, comme en Egypte (Semmoud, Denis, 2001 ; Deboulet 2000a, Séjourné 2006), en Tunisie (Legros 2003), en Jordanie (Ababsa, à paraître) le raccordement aux infrastructures de base constitue un moyen de régulation sociale, en régularisant de facto -et pas toujours de jure- la tenure foncière dans les quartiers non-réglementaires. De surcroit, les gouvernements eux-mêmes, plutôt que les collectivités locales, ont dans ces cas la haute main sur ces opérations où elles engagent leur légitimité. L'augmentation des tarifs, le renchérissement des couts de raccordement peuvent dans ce contexte représenter des motifs de contestation sociale dont les gouvernements se défient. Toutefois, la sensibilité populaire n'est souvent pas la même d'un secteur à l'autre. Alors que l'eau potable constitue certainement le secteur le plus sensible, pour des raisons symboliques autant qu'économiques, l'électricité et surtout le secteur des déchets sont généralement perçus comme moins problématiques. Le coup de frein sur les grands contrats de délégation ou même de gestion pour la distribution d'eau potable peut sans doute s'expliquer par le souci de réduire la contestation alors que les PPP dans le domaine des déchets en Egypte (Florin 2010*); en Jordanie (Debout, à paraître) ou en Tunisie (Rebhi 2010 ; Tizaoui 2009) ou plus ponctuellement dans le domaine de l'électricité (comme en Jordanie, Verdeil 2010) ont fait florès. Pour autant, à l'épreuve des faits, ces secteurs peuvent aussi être la cible de puissants 
mouvements de contestation, comme le montre Florin dans ce dossier à propos des réformes de la collecte des ordures ménagères au Caire. Une question complémentaire, à laquelle les éléments réunis ici ne permettent pas de répondre, serait de savoir si certaines villes (les capitales notamment) ou certaines régions (d'opposition ou au contraire, fidèles au régime), constituent des enjeux plus stratégiques que d'autres dans la mise en place et la régulation des réformes des services.

\section{Réformes des services urbains et réformes territoriales : des articulations plurielles}

Si le partenariat avec des partenaires privés n'est donc pas si souvent la voie choisie par les autorités publiques pour réformer les services publics essentiels, une autre méthode consiste à agir sur l'échelon territorial de gestion de ces services. La thématique de la décentralisation est en effet, aux côté de la promotion des PPP, un des mots d'ordre des réformes institutionnelles véhiculées par les grands bailleurs de fonds internationaux. Toutefois, dans la pratique, et en particulier dans les pays arabes, il est bien difficile d'identifier une articulation univoque entre réforme de la gestion des services urbains et décentralisation. Certes, dans un pays comme le Maroc, les communes ont acquis d'importantes prérogatives dans ce domaine, comme le rappelle de Miras (2010*). Mais dans les autres pays de la région, les politiques de décentralisation sont généralement à peine ébauchées (Le Bris, 2004) et en réalité, les transformations en cours varient entre permanence de la gestion centralisée, déconcentration (gestion localisée d'un service restant sous la tutelle étatique) et recentralisation.

L'exemple de la réforme des déchets au Caire, analysée par Florin (2010*), est un bon exemple d'une réforme qui s'effectue à une échelle de gestion inchangée, puisque ce sont les gouverneurs qui sont en charge de son pilotage. Toutefois, Lise Debout (2010) montre que d'un gouvernorat à l'autre, d'importantes variations peuvent se produire. Sans réforme territoriale, des différentiations dans la gestion des services publics ne sont donc pas toujours exclues. On retrouve ce schéma au Liban à propos de la gestion de l'électricité. Malgré l'existence d'une entreprise publique nationale, théoriquement garante d'un fonctionnement uniforme sur tout le territoire, d'importantes différentiations des modes de gestion se produisent d'une région à l'autre, pour tenir compte des rapports de force sociaux et politiques locaux (Verdeil, 2009 ; Gabillet 2010*).

Du reste, dans un même pays mais pour un secteur différent, les pratiques peuvent varier. Ainsi, au Liban, l'Etat appuyé par des bailleurs de fonds (Banque mondiale, Agence française de développement) mène depuis la fin des années 1990 une importante réforme du secteur de l'eau. Celle-ci combine un double mouvement de déconcentration et de recentralisation. En effet, le ministre de l'Eau délègue ses compétences de gestion à des établissements publics régionaux qui restent sous sa tutelle, avec une autonomie financière. Mais en pratique, ces établissements, au nombre de 4, regroupent les 26 offices des eaux préexistants à l'échelle des cazas (département libanais). En tout état de cause, les municipalités restent marginales dans ce dispositif, voire sont dépouillées des compétences qu'elles détenaient dans le domaine de l'assainissement : il y bien recentralisation. Christèle Allès (2010*) identifie une autre caractéristique intéressante à propos de l'établissement des eaux du Liban-Sud, qui semble illustrer la capacité d'une administration publique réformée à s'affirmer face à des évolutions néolibérales censées miner l'action de l'administration étatique. Dans un contexte politique peu favorable de désengagement de l'Etat de la politique de reconstruction et dans le contexte d'une politique de réforme initialement orientée vers des PPP, cet 
établissement public parvient globalement à imposer son autorité et ses projets face aux acteurs politiques locaux, et à instaurer des relations relativement équilibrées avec les ONG internationales qui gèrent massivement les capitaux de la reconstruction d'après la guerre de 2006.

Le dernier exemple présenté dans ce dossier est la réforme du secteur de l'eau dans les territoires palestiniens. Ici, c'est une configuration inverse qui prévaut (Signoles, 2010*). La distribution de l'eau (et par ailleurs, de l'électricité) est historiquement l'apanage des municipalités, seules institutions palestiniennes réellement puissantes dans le contexte de l'occupation des territoires occupés. Les réformes d'inspiration libérale des années 1990 vont ici de pair avec le souci de construire un centre étatique, au détriment des municipalités et parallèlement, en récupérant des prérogatives détenues par les Israéliens. L'analyse de la première phase de ces réformes (jusque vers 2004-2005) montre, dans le contexte politique de l'Intifada défavorable à l'affirmation de l'Autorité palestinienne, la résistance des municipalités et des pouvoirs locaux à la mise en œuvre de la réforme et leur capacité à la modifier voire à la bloquer.

L'introduction de la dimension territoriale dans l'analyse de ces réformes permet d'aller au-delà de l'opposition entre gestion publique et gestion privée, tout en prenant en compte d'autres acteurs et d'autres intérêts sociaux qui pèsent fortement sur l'évolution de secteurs très fortement inscrits dans l'espace local (en particulier l'eau et les déchets).

\section{La place des petits opérateurs privés dans la gestion urbaine}

La recherche sur les services urbains est renouvelée par le nouveau regard porté sur le petit secteur marchand, autrefois dénommé informel. Les petits opérateurs privés (POP) font figure de solution innovante, en articulation avec les grands opérateurs publics ou privés, aux difficultés de l'intervention dans les quartiers pauvres. Leur réactivité et leur flexibilité (y compris sur le plan social), figurent au nombre de leurs atouts, par opposition aux lourdeurs de gestion des opérateurs officiels. Toutefois, à la différence des classiques petits revendeurs d'eau, les POP apparaissent aussi comme des entreprises dotées d'une technicité plus importante, capable de proposer des solutions de desserte proches des opérateurs classiques, en termes techniques et en qualité. Par exemple, au Vietnam, les POP assurent une desserte domiciliaire en eau potable. De surcroit, et dans cet exemple, ce service bénéficie d'une reconnaissance officielle avec l'opérateur officiel, même si les promesses initiales se révèlent parfois, dans la durée, quelque peu décevantes (Botton, Blanc, 2010). Dans quelle mesure peut-on identifier l'émergence d'une telle catégorie d'acteurs dans les pays arabes et comment les politiques publiques la prennent-elles alors en compte?

Peu de travaux ont abordé directement cette question, ce qui reflète probablement l'état de la réflexion parmi les autorités en charge des services urbains. Toutefois, quelques expériences sont signalées dans la littérature même si elles ne sont pas catégorisées sous le label POP. Ainsi, Aziz Iraki et Mohamed Tammim (2009) décrivent dans les périphéries de Casablanca des associations locales de résidents qui parviennent à construire et à gérer des bornes fontaines, dans une sorte de soustraitance avec la Lydec $^{6}$. Mais il ne s'agit pas ici d'un service techniquement aussi poussé que dans les exemples signalés ci-dessus, dans la mesure où aucune desserte domiciliaire n'est proposée et ces associations n'ont pas de structure d'entreprise ni de vocation capitaliste.

\footnotetext{
${ }^{6}$ Lyonnaise des eaux de Casablanca, concessionnaire du service d'eau potable.
} 
Deux autres exemples pris dans ce dossier se rapprochent de ces POP. Au Caire, la contribution essentielle des chiffonniers (zabbâlîn) à la collecte des déchets a été reconnue par l'octroi de licences (payantes) depuis 1980. Toutefois, ce système a été remis en cause avec les réformes introduites en 2000 et les délégations de service octroyées à des entreprises privées d'abord uniquement étrangères puis aussi égyptiennes. Néanmoins, B. Florin signale ${ }^{7}$ l'existence de contrats de soustraitance au profit d'entreprises créées par certains chiffonniers. Cette situation reste limitée à des périmètres particuliers et très liée à la stratégie propre des entreprises. Elle s'inscrit par ailleurs dans un système global de déni des compétences des travailleurs du secteur informel et des services qu'ils rendent. A cet égard, cet exemple ne s'apparente pas du tout à l'expérience des POP.

L'exemple des générateurs électriques dans les villes libanaises, finement étudié par P. Gabillet $\left(2010^{*}\right)$ est également intéressant à considérer dans cette perspective. Face à la pénurie d'électricité, des petits entrepreneurs ont développé de véritables réseaux électriques à l'échelle des quartiers ou de petites villes, desservant les abonnés à leur domicile. On constate même, dans l'exemple de Jbeil, des investissements d'entreprises capitalistes. Selon une étude de la Banque mondiale, leur puissance installée représenterait $38 \%$ de celle de l'entreprise publique. Mais le paradoxe de cette situation est que ces petits opérateurs privés ne sont que tolérés par les autorités publiques, dans l'attente d'une hypothétique amélioration du système de production officiel. Aucun partenariat ne les lie donc à l'opérateur public. P. Gabillet met toutefois en évidence, à l'échelle locale, des tentatives de régulation par les autorités municipales, sans toutefois qu'émerge un cadre réglementaire. En fait, leur activité est fortement dénigré par les autorités, notamment pour des raisons sanitaires (la production de ces générateurs étant très polluante : voir Dagher, Ruble, 2010) et de sécurité sur les réseaux. Leurs coûts de production sont également considérés comme peu rentables par rapport à ce que permettrait une augmentation de la production électrique classique. Dans le domaine de l'électricité, les solutions non-conventionnelles et décentralisées généralement mises en avant reposent plutôt, il est vrai, sur la mobilisation de ressources renouvelables (solaires, éoliennes) même si jusqu'à présent ce modèle ne semble encore guère avoir fait ses preuves pour une production et une desserte de masse dans les pays du Nord, et à fortiori au Sud (Coutard et Rutherford, 2009).

Dans ces deux exemples, à des degrés différents, et au-delà des spécificités techniques propres à chaque secteur, les petites entreprises privées semblent faire face à un véritable déni par rapport aux solutions conventionnelles et industrielles, liées à un savoir-faire technique poussé et associées à des modes de fonctionnement hiérarchique (top down) et à une esthétique de l'espace urbain laissant peu de place à l'informel et à ses bricolages. Il y a là cependant un enjeu qui doit faire l'objet d'analyses plus approfondies.

\section{Conclusion}

Ce dossier sur les réformes des services urbains dans les pays arabes laisse entrevoir, à travers les quelques exemples présentés, d'une représentativité forcément limitée, des évolutions spécifiques mais aussi quelques traits communs avec les tendances globales.

\footnotetext{
${ }^{7}$ En s'appuyant entre autres sur les travaux de Lise Debout. Dans l'attente de la thèse en cours de rédaction de cette dernière, on pourra consulter son étude pour l'AFD (Debout 2010).
} 
Un véritable décalage, en termes quantitatifs et chronologique, apparaît par rapport à d'autres régions du monde. Globalement, la vague des « privatisations » a été ici beaucoup moins forte qu'ailleurs (au moins pour l'eau et l'électricité). Si l'on ne dispose pas d'indicateurs pour cerner les réformes du secteur public, les exemples réunis et ceux dont la littérature se fait l'écho semblent indiquer des évolutions relativement plus contrôlées qu'ailleurs également. Il est tentant de mettre cette orientation en lien avec la persistance d'Etats autoritaires, dont le monde arabe n'a certes pas le monopole, mais où il se combine avec un modèle d'économie rentière ou semi-rentière (Catusse 2006). Les services publics et en particulier les services urbains en réseau, symbole de l'accès à la modernité, y constituent un élément central du pacte social entre les Etats et leurs citoyens. Les remises en question de ce modèle sont potentiellement lourdes de contestations politiques, qu'illustrent les contestations de la réforme des déchets au Caire, où les émeutes électriques au Liban en 2008 (Verdeil, 2009) et qui sont craintes par les Etats. C'est là sans doute une question à creuser.

Si la reconnaissance et la promotion des petits opérateurs marchands est encore loin de constituer une norme mondiale, les exemples tirés de ce dossier suggèrent que sur ce point aussi, le monde arabe se distingue, du moins pour les services étudiés ici (ce n'est pas le cas pour les transports). Faut-il y voir le reflet de situation d'accès moins dramatique qu'ailleurs ? Ou la réticence d'administrations et d'élites politiques rechignant à déléguer leurs prérogatives et défiantes des innovations allant à l'encontre des techniques conventionnelles modernes ? Des compléments d'information sont également souhaitables sur ce point.

Au-delà des destinées contrastées des réformes de ces différents secteurs, imbriquant partenariats public-privé, réformes territoriales et accords avec des acteurs informels, un effet commun de leur mise en œuvre (et parfois en défaut) ressort, qui fait en revanche le lien avec des observations faites ailleurs, par exemple en Afrique subsaharienne (Jaglin 2005). Leur inachèvement et la multiplication des expérimentations se traduit par une différentiation territoriale entre villes (Gabillet, 2010*) mais aussi, à l'échelle d'une même ville, par exemple au Caire (Florin 2010*). Cette différentiation passe par la disparité croissante des assemblages et dispositifs gestionnaires, sur le plan technique (reflétant la diversité des clientèles ou des prestataires) mais aussi institutionnel et politique. Dans des pays où les réformes de décentralisation sont restées globalement très timides (avec là encore, une relative exception marocaine) (Signoles, 2006 ; Le Bris et alii, 2004), ce résultat sonne comme un paradoxe. Sans doute peut-il s'analyser comme un signe de la néolibéralisation de l'espace (Peck, Tickell, 2002), faisant cohabiter zones exclusives gérées par le marché, zones informelles maintenues sous l'épée de Damoclès de l'arbitraire étatique et des mafias et gouvernance autoritaire, dont Le Caire décrit par Singerman constitue un exemple emblématique (dont rend compte ici Barthel, 2010). Cette complexification territoriale rend particulièrement difficile une appréhension unifiée de la situation des services urbains comme elle réduit à néant les espoirs d'identifier ne serait qu'un ensemble unifié de recettes et d'interventions venant d'en haut et ne prenant pas compte les spécificités des villes et des populations considérées, tout comme leurs ressources et leurs désirs.

Bibliographie

ABABSA, à paraître, "La gestion des quartiers informels en Jordanie : de la réhabilitation à la seule provision de services (1980-2007) », in Puig N. \& Doraï M. K. (dirs.), L'urbanité des marges. Migrants, réfugiés et relégués dans les villes du Proche-Orient 
ALLAIN-EL MANSOURI B., 2001, L'Eau et la Ville au Maroc. Rabat-Sale et sa périphérie, L'Harmattan, $254 \mathrm{p}$.

Allès 2010

BAKKER K., 2003, « Archipelagos and networks: urbanization and water privatization in the South », The Geographical Journal, vol. 169, p. 328-341.

BAKKER K., 2007, « Trickle Down? Private sector participation and the pro-poor water supply debate in Jakarta, Indonesia », Geoforum, vol. 38, n. 5, p. 855-868.

BAKKER K., 2009, « Participation du secteur privé à la gestion des services des eaux : tendances récentes et débats dans les pays en voie de développement », Espaces et sociétés, vol. 139, n. 4, p. 91.

BARTHEL P.A., 2010, «Cairo Contested. Governance, Urban Space and Global Modernity, D.

Singerman ed. ", Géocarrefour, ce numéro

BOTTON S. ET BLANC A., 2010, Accès de tous aux services d'eau : le rôle des petits opérateurs privés à Hô Chi Minh Ville, Vietnam, Paris, AFD, coll. «Focales», 102p.

CATUSSE M., 2006, « Ordonner, classer, penser la société : les pays arabes au prisme de l'économie politique », dans Elizabeth Picard (dir.) La politique dans le monde arabe, Armand Colin, p. 215-238.

COUTARD O. et RUTHERFORD J. (éd.), 2009, « Aux marges des réseaux », Flux, nº76, 168p.

COUTARD Olivier, 2008, « Placing splintering urbanism: Introduction », Geoforum, vol. 39, $\mathrm{n}^{\circ}$. 6, p. 1815-1820.

DAGHER L. et RUBLE I., 2010, « Challenges for CO2 mitigation in the Lebanese electricpower sector », Energy Policy, vol. 38, n. 2, p. 912-918.

DARMAME K. et POTTER R. B., 2009, « Gestion de la rareté de l'eau à Amman : rationnement de l'offre et pratiques des usagers », Espaces et sociétés, vol. 139, nº. 4, p. 7189.

DE MIRAS 2010 ce numéro

DEBOULET A., 2000, « Accéder à l'eau, dans un contexte d'ajustement, en milieu urbain égyptien ", Les Cahiers d'URBAMA, n. 15, p. 61-78.

DEBOULET A., 2000, L'eau en milieu urbain dans le monde arabe, Tours, URBAMA, 119 pp. Debout L., à paraître,

Debout L., 2010, Partenariats public-privé et gestion locale des déchets ménagers en Egypte, Rapport final de l'étude réalisée dans le cadre de la convention RICH n¹40-2009, Agence française de développement, $50 \mathrm{p}$.

DURAND-LASSERVE A., 2004, « La question foncière dans les villes du Tiers-monde : un bilan ", Economies et sociétés, vol. 42, n ${ }^{\circ}$. , Série Développement, croissance et progrès, p. 1183-1211. 


\section{FLORIN 2010}

\section{GABILLET 2010 (dans ce $n^{\circ}$ )}

GASSNER K. et POPOV A.et PUSHAK N., 2009, Does private sector participation improve performance in electricity and water distribution?, World Bank - PPIF, coll. «Trends and Policy Options», 118p, accédé 26 octobre 2009, à

http://www.pdfdownload.org/pdf2html/view_online.php?url=http\%3A\%2F\%2Fwww.ppiaf.or g\%2Fdocuments\%2Ftrends_and_policy\%2FPSP_water_electricity.pdf.

GERLACH E. et FRANCEYS R., « Regulating water services for the poor: The case of Amman », Geoforum, vol. 40, n. 3, 2009, p. 431-441.

GODARD X., 2002, Les Transports et la Ville en Afrique au sud du Sahara, Karthala, 408 p.

GRAHAM S. et MARVIN S., 2001, Splintering urbanism : networked infrastructures, technological mobilities and the urban condition, London, Routledge, XXIX-479p.

IRAKI A. et TAMIM M., 2009, « Organisation des populations et place des élites locales dans la gestion politique d'une périphérie de Grand Casablanca : le cas de Dar Bouaza (Maroc) », dans A. Iraki, R. Cattedra, O. Legros, P. Signoles (dir.) Les effets territoriaux des politiques publiques dans les périphéries urbaines. Exemples au Maghreb, Tours, CITERES, coll. «Les Cahiers d'EMAM», p.85-114.

JAGLIN S., 2005, Services d'eau en Afrique subsaharienne : la fragmentation urbaine en question, Paris, CNRS éditions, 244 p.

JAGLIN, ZERAH, à paraître, Réforme des services publics en réseaux: où en est-on dans les villes en développement ? (titre sous réserve), Dossier pour la Revue Tiers Monde (2010 ?)

LE BRIS Emile, 2004, Les municipalités dans le champ politique local : les effets des modèles exportés de décentralisation sur la gestion des villes en Afrique et au Moyen-Orient, Programme de recherche urbaine, MAE-ISTRED-GEMDEV, X p.

LEGROS O., 2003, Le gouvernement des quartiers populaires : production de l'espace et régulation politique dans les quartiers non réglementaires de Dakar (Sénégal) et de Tunis (Tunisie), Université François Rabelais, Tours, 485 p.

MARTINAND C. (éd.), 2009, Eau \& assainissement des villes et pays riverains de la Méditerranée, Paris, IPEMED, 61p, accédé 28 mai 2010, à http://www.ipemed.coop/spip.php?article264.

MIRAS DE C. LE TELLIER J., 2005, Gouvernance urbaine et accès à l' eau potable au Maroc, Editions L'Harmattan, 275 p.

PECK J. et TICKELL A., « Neoliberalizing Space », Antipode, vol. 34, nº. 3, 2002, p. 380404.

REBHI A., 2010, « Les services urbains de la régie municipale à la gestion déléguée : l'exemple de ramassage des déchets des ordures ménagers à Kairouan (Tunisie) ", communication au séminaire TANMIA La fabrique du développement dans le monde arabe, Genève, 18-19 mars. 
SCHNEIER-MADANES G. ET GOUVELLO B. de (éd.), 2003, Eaux et réseaux : les défis de la mondialisation, Paris, IHEAL, coll. «Travaux et mémoires de l'IHEAL, 346p.

SEJOURNE M., 2006, Les politiques récentes de "traitement" des quartiers illégaux au Caire : nouveaux enjeux et configuration du système d'acteurs?, Université François Rabelais de Tours.

SEMMOUD B. et DENIS E., 2001, « De l'inégalité d'accès aux services publics "marchands" à la question de l'environnement. A propos de l'eau en Egypte », dans Chantal ChansonJabeur et alii (dir.) Les services publics et leurs dynamiques au Machreq et au Maghreb : actes du colloque organisé du 19 au 21 mai 1999 à Paris, Paris, L'Harmattan, p. 129-153.

SEMMOUD B., « En guise de synthèse: Les services publics entre mondial et local. L'exemple du Maghreb et du Moyen-Orient », dans Chanson-Jabeur Chantal et alii, Les services publics et leurs dynamiques au Machreq et au Maghreb : actes du colloque organisé du 19 au 21 mai 1999 à Paris, Paris, L'Harmattan, 2001, p. 659-682.

SIGNOLES A., 2006, « Réformes de l'Etat et transformation de l'action publique », dans Elizabeth Picard (dir.) La politique dans le monde arabe, Armand Colin, p. 239-262.

TIZAOUI H., 2009 « Privatisation du service de la collecte des ordures ménagères en Tunisie. La "Good Governance" ou la mise à niveau des villes tunisiennes, étude de cas: La commune de Hamman el Chott ", dans Jean-Marie Miossec (dir.) Terrains et échelons de la gouvernance : expériences en France et au Maghreb, Paris, L'Harmattan, p. 231-265.

TOMAIRA R. V., 2008, Legacy of a Rentier State: Reforming Jordan's Water, Energy and Telecommunications Sectors, PhD Regional and Urban Studies, Berkeley, University of California, $248 \mathrm{p}$.

VERDEIL É., « Electricité et territoires : un regard sur la crise libanaise », Revue Tiers Monde, vol. 198, 2009, p. 421-438.

VERDEIL É., « Libéralisation, nouvelle gouvernance locale et développement urbain durable : stratégies jordaniennes face à la dépendance énergétique », Hammamet, 2010.

YOUSFI H., Le contrat dans une coopération internationale. La rencontre des intérêts à l'épreuve de la rencontre des cultures. Cas du Contrat de gestion déléguée de l'Alimentation en Eau potable de la Communauté Urbaine de Tripoli (Liban), 2006, Paris X-Nanterre, 313 p. 